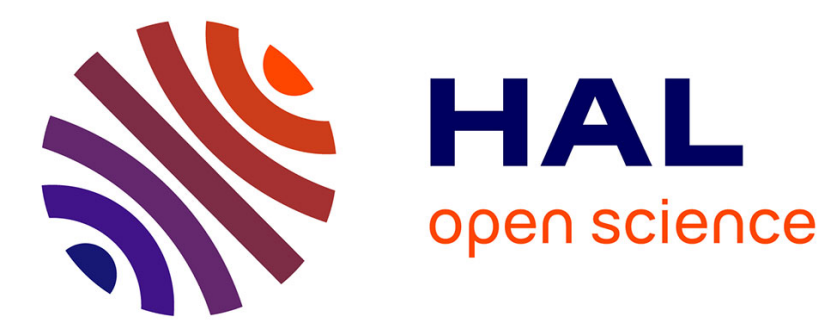

\title{
La pollution atmosphérique particulaire sous la surveillance du lidar \\ Patrick Chazette
}

\section{To cite this version:}

Patrick Chazette. La pollution atmosphérique particulaire sous la surveillance du lidar. Clefs CEA, 2009, Terre et Environnement, 57, pp.75-79. hal-03325955

\section{HAL Id: hal-03325955 \\ https://hal.science/hal-03325955}

Submitted on 25 Aug 2021

HAL is a multi-disciplinary open access archive for the deposit and dissemination of scientific research documents, whether they are published or not. The documents may come from teaching and research institutions in France or abroad, or from public or private research centers.
L'archive ouverte pluridisciplinaire HAL, est destinée au dépôt et à la diffusion de documents scientifiques de niveau recherche, publiés ou non, émanant des établissements d'enseignement et de recherche français ou étrangers, des laboratoires publics ou privés. 


\section{La pollution atmosphérique particulaire sous la surveillance du lidar}

La technique du lidar, telle qu'elle a été adaptée par des chercheurs du CEA et du CNRS, constitue un outil de choix pour le contrôle de la pollution de l'atmosphère par des particules. L'émission d'un faisceau laser vers la zone à étudier puis l'analyse de sa rétrodiffusion par les molécules et les particules permet d'en déterminer la densité, la localisation et même la nature ou la géométrie.

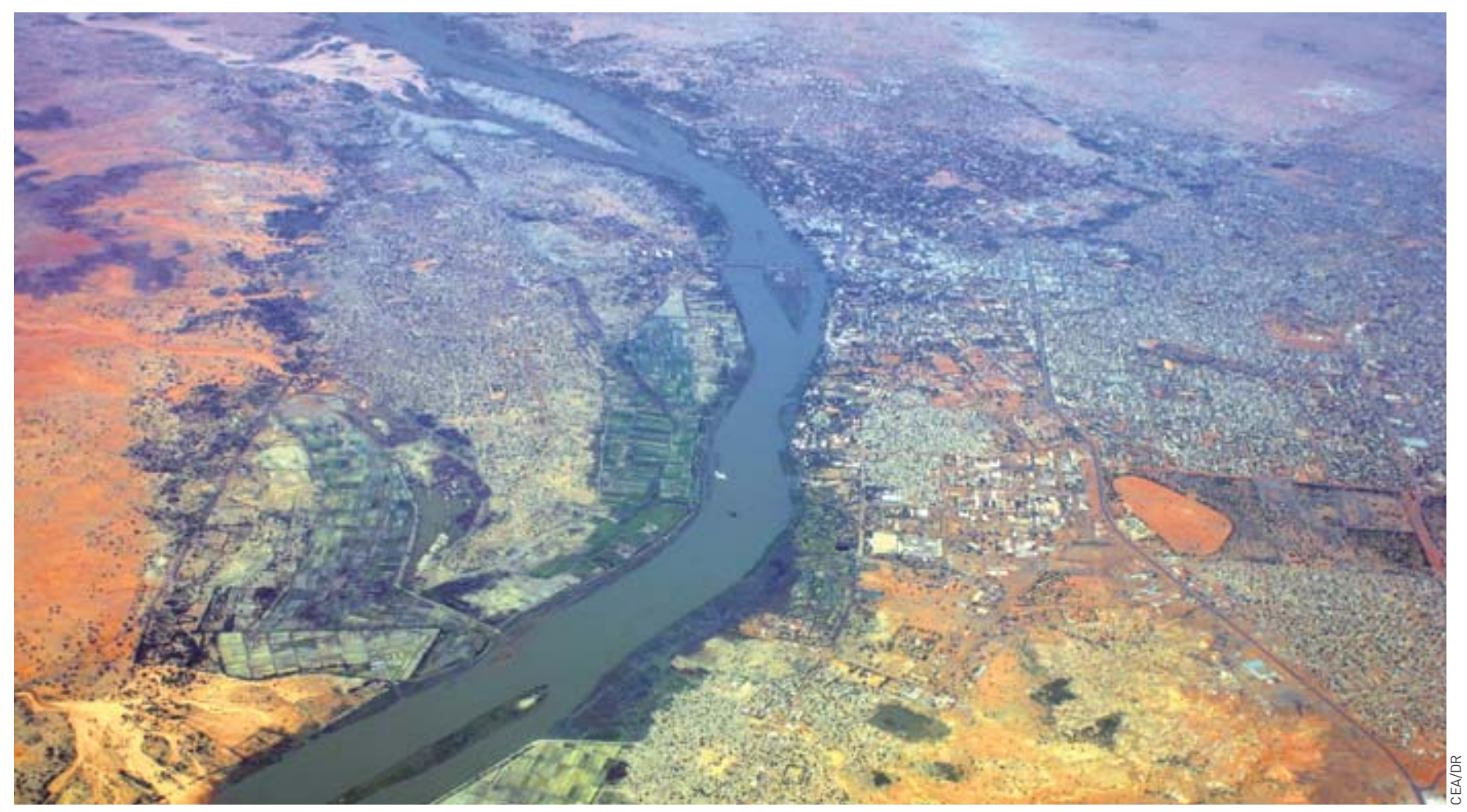

e lidar aérosol ultraviolet aéroporté (Lauva) dési— gne un système Light Detection and Ranging (lidar) conçu conjointement par le CNRS et le CEA. Finalisé, en 2004, cet instrument a pour mission d'étudier et de suivre l'évolution de la qualité de l'air. Le terme "aéroporté" a été ajouté au sigle, en raison de la compacité du système et donc de son adaptation à l'embarquement à bord de petits aéronefs. La société Leosphere ${ }^{(1)}$ en assure la commercialisation sous le nom d'Ez-lidar ${ }^{\circledR}$ avec une licence du CEA et du CNRS. Conforme aux normes de sécurité oculaire, il peut s'utiliser sans risque dans les régions habitées.

\section{Contexte sociétal}

Le système lidar Lauva a été initialement développé pour répondre à des problématiques scientifiques d'actualité liées à l'impact environnemental des par-

(1) Pour en savoir plus sur cette société: www.leosphere.com ticules émises dans l'atmosphère du fait des activités humaines.

En effet, les particules anthropiques peuvent polluer notre environnement de trois manières différentes. D'abord par leur action sur la santé des populations en induisant des insuffisances respiratoires voire des pathologies cardiovasculaires. Parmi les groupes de population les plus sensibles et donc les plus menacés figurent les jeunes enfants, les personnes asthmatiques et naturellement les personnes âgées. Ensuite, les particules anthropiques interagissent avec le rayonnement solaire en diminuant la visibilité et en modifiant l'équilibre énergétique du système couplé Terreatmosphère. Cette interaction, si elle conduit à un refroidissement de la surface et va dans le sens inverse de l'impact climatique des gaz à effet de serre, induit aussi et simultanément un réchauffement de la couche d'atmosphère où nous vivons. Le refroidissement de la surface se trouve accentué par le rôle que les particules anthropiques jouent dans la formation

Survol de Niamey en ULM. 
des nuages. En provoquant une diminution de la taille des gouttelettes d'eau, ces particules accentuent l'efficacité avec laquelle les nuages vont renvoyer la lumière du Soleil vers l'espace ainsi que la durée de vie des nuages dans l'atmosphère. Enfin, les particules anthropiques entraînent également une modification du cycle de l'eau et des ressources en eau potable de certaines régions du globe, notamment les régions sahéliennes. Elles proviennent alors de la destruction intensive de la savane et de la forêt tropicale par le feu (par exemple, pour l'écobuage).

Pour comprendre et prévoir toutes les implications environnementales des particules anthropiques, les mesures en surface demeurent insuffisantes. D'où l'impératif de connaître leur répartition verticale ainsi que l'évolution temporelle de cette dernière. Par exemple, les concentrations rencontrées en un lieu donné d'une ville ne se trouvent pas seulement dépendantes des émissions locales car les polluants peuvent être transportés en altitude et redistribués en surface.
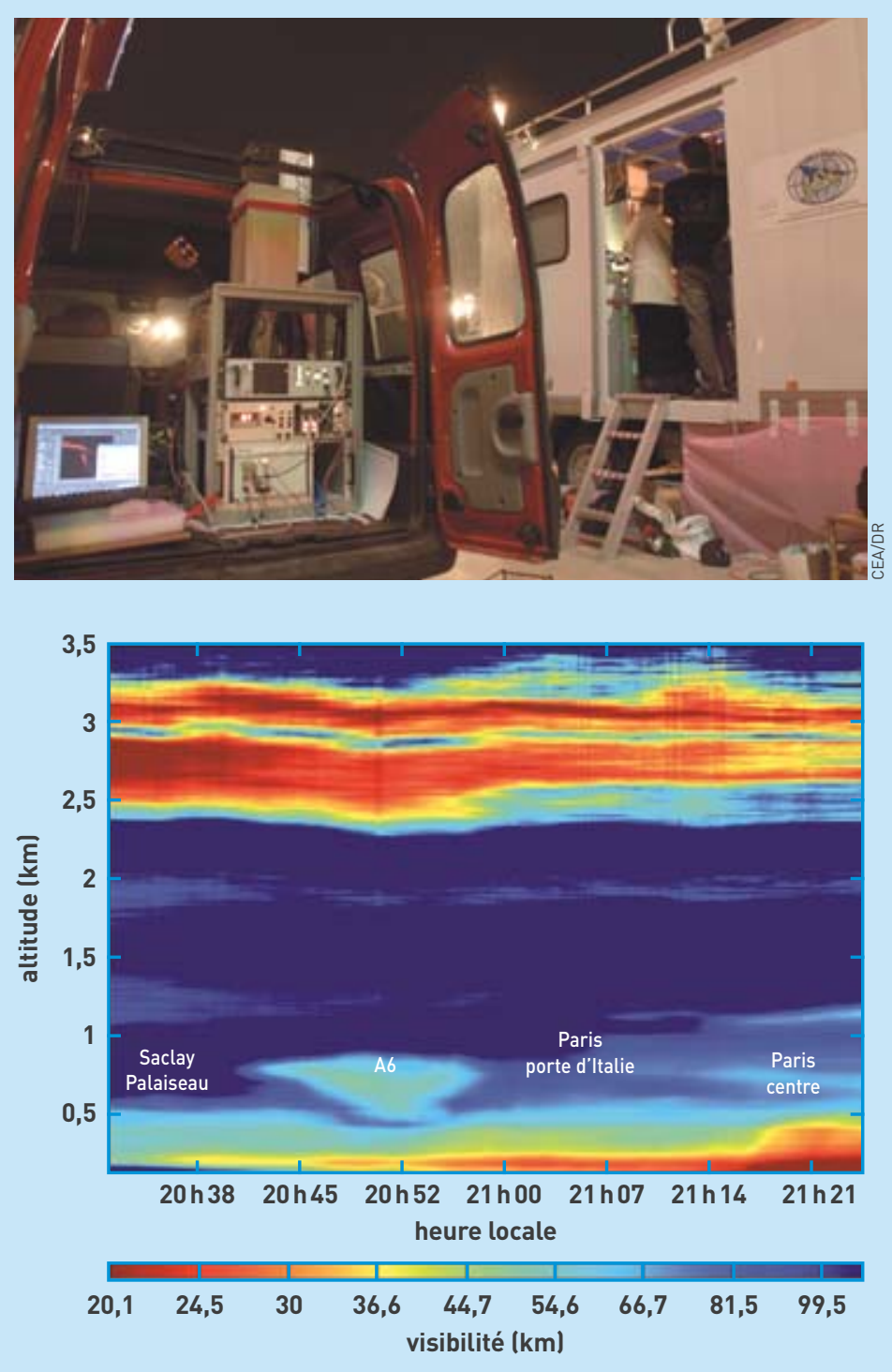

Figure 1.

Système lidar prototype développé par le CEA et le CNRS embarqué dans une voiture de service du CEA pour l'étude de la qualité de l'air sur Paris. Des mesures ont été effectuées entre la banlieue Sud (Saclay) et le centre de Paris. Un exemple est donné ici pour la visibilité déduite de l'observation lidar.
Dans les agglomérations occidentales, la norme sanitaire pour la pollution particulaire s'établit à partir des mesures, effectuées en surface, de la concentration massique (dite PM10) pour les particules de diamètre aérodynamique inférieur à $10 \mu \mathrm{m}$. Ces particules sont susceptibles de pénétrer dans notre système respiratoire, et cela plus profondément pour celles inférieures à $2,5 \mu \mathrm{m}(\mathrm{PM} 2,5)$ et même $1 \mu \mathrm{m}$ (PM1). Depuis 1999, une directive européenne ${ }^{(2)}$ a fixé les seuils de polluants particulaires présents à la surface: elle a été renforcée depuis les récents événements de pollution particulaire associés aux périodes de canicule. Ceci a conduit la France à élaborer une circulaire, en date du 12 octobre 2007, relative à l'information du public sur les particules en suspension dans l'air ambiant. Cette information intervient dès le dépassement, en moyenne journalière, du seuil de $80 \mu \mathrm{g} / \mathrm{m}^{3}$.

Le système lidar, développé par le CEA et le CNRS, permet de connaître la répartition verticale des polluants particulaires avec une résolution de 1,5 m entre la surface et la tropopause (vers $10-12 \mathrm{~km}$ d'altitude). La longueur d'onde utilisée, de $355 \mathrm{~nm}$, est sensible aux particules submicroniques (PM1) qui composent pour plus de $99 \%$ les aérosols de pollution.

\section{La surveillance de la qualité de l'air des mégalopoles}

En mai 2005, les premiers tests du système ont été effectués à l'occasion du programme lidar pour la surveillance de l'air (Lisair) soutenu par la mairie de Paris. Deux systèmes lidar, couplés à l'observation in situ, furent utilisés pour cette étude: le premier situé sur l'esplanade de l'Hôtel de Ville en station fixe et le

(2) Directive $\mathrm{n}^{\circ}$ 1999/30/CE du 22 avril 1999.

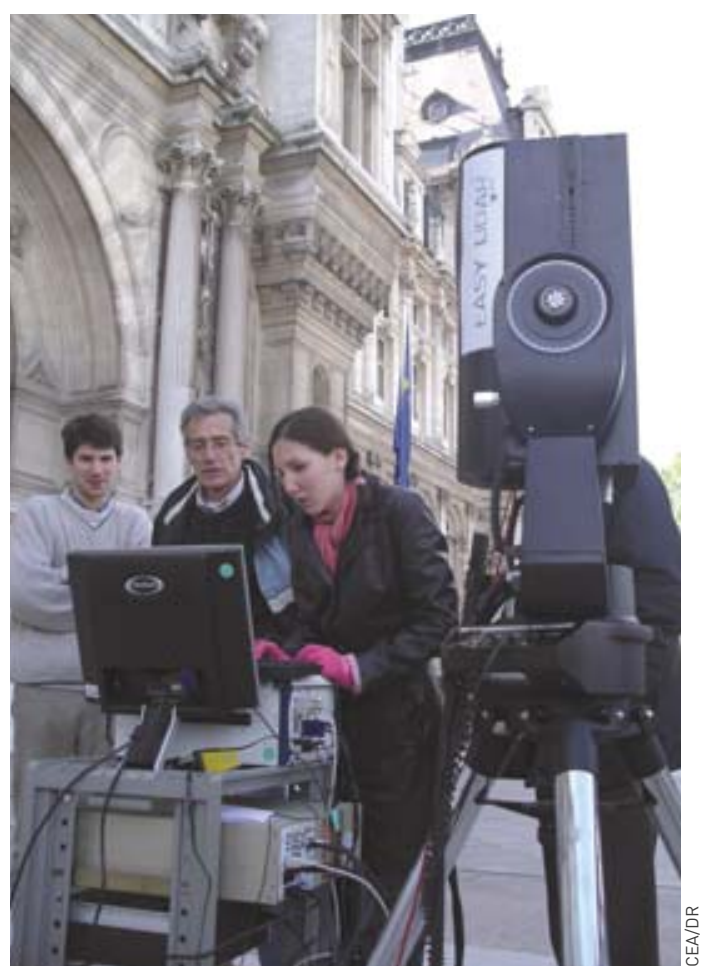

Système Lauva au sol installé sur le parvis de l'hôtel de ville de Paris. 


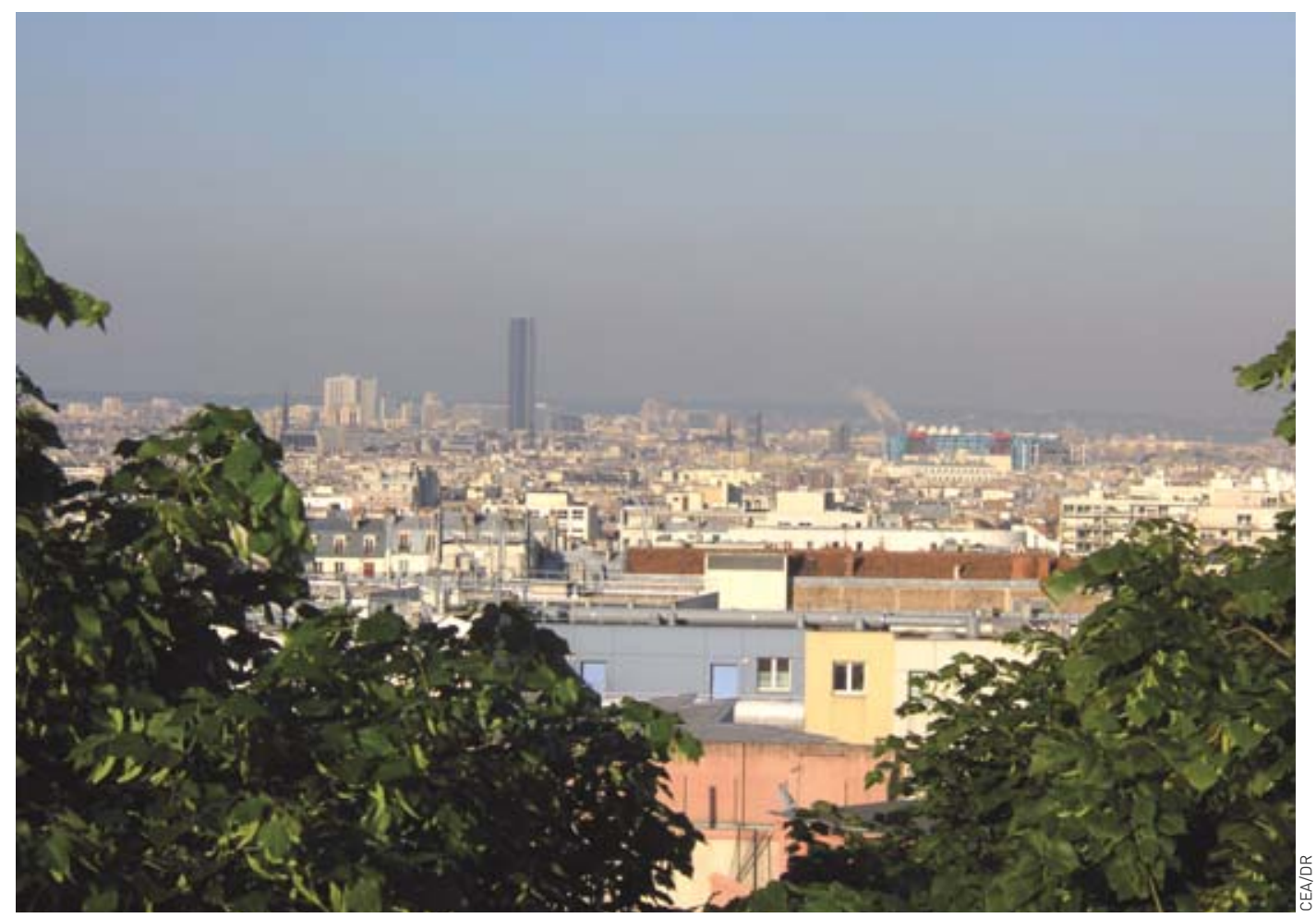

Pollution sur Paris.

second embarqué à bord d'une voiture de service du CEA (figure 1). Cette approche expérimentale a permis de caractériser les particules de pollution émises par la circulation automobile, via leur taille, leur composition chimique et leurs propriétés optiques. Leur évolution dans la colonne d'air a été suivie à la fois dans l'espace et le temps. Ce fut le cas, notamment, le 26 mai 2005, avec des mesures réalisées au centre de Paris et dans la banlieue Sud (Saclay). La capitale se trouvait alors exposée à des masses d'air chargées en particules provenant des feux de forêts sévissant en Espagne et au Portugal et dont le panache s'éleva jusqu'à 2,5 et même $3,5 \mathrm{~km}$ d'altitude. En dessous de $1 \mathrm{~km}$, la pollution locale est apparue avec des visibilités plus faibles vers le centre de Paris. Des mesures ayant été effectuées en soirée, la création d'une couche réservoir de polluants fut mise en évidence au-dessus de l'autoroute A6, entre 0,5 et $1 \mathrm{~km}$. Cette couche réservoir aurait pu être transportée audessus d'autres régions ou se déverser le matin sur Paris en cas de vent faible, comme cela a été le cas. Plus tard dans la soirée, la qualité de l'air sur Paris fut influencée par les particules de feux dont certaines atteindront la rue.

Suite à la campagne Lisair, la société Leosphere et le CEA ont initié leurs premiers contacts avec la Chine pour valoriser le système lidar à l'international. Une première démonstration a été réalisée sur le toit de l'Institut de physique atmosphérique de Pékin (figure 2). En surface, la visibilité y est, en moyenne,

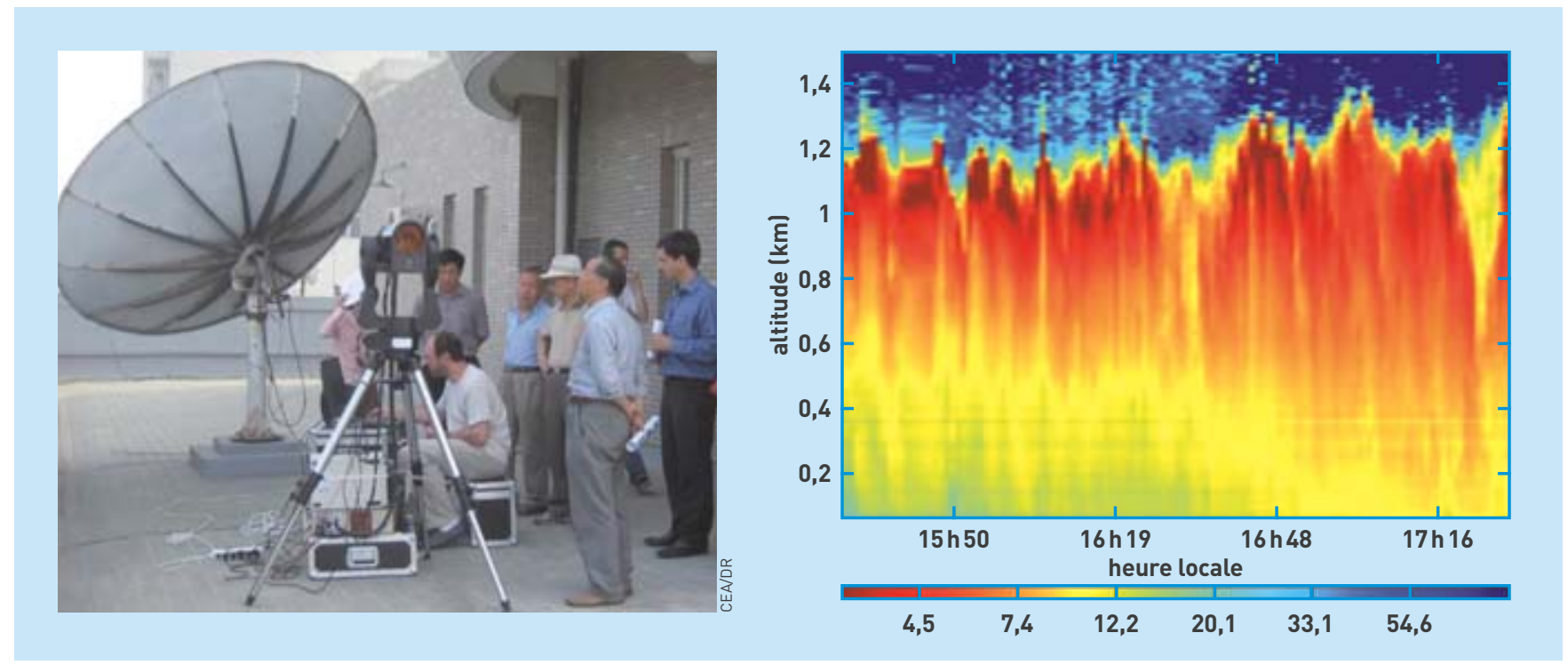

Figure 2.

Système lidar sur le toit de l'École polytechnique de Pékin. Les mesures de visibilité déduite de l'observation lidar sont données à droite. 


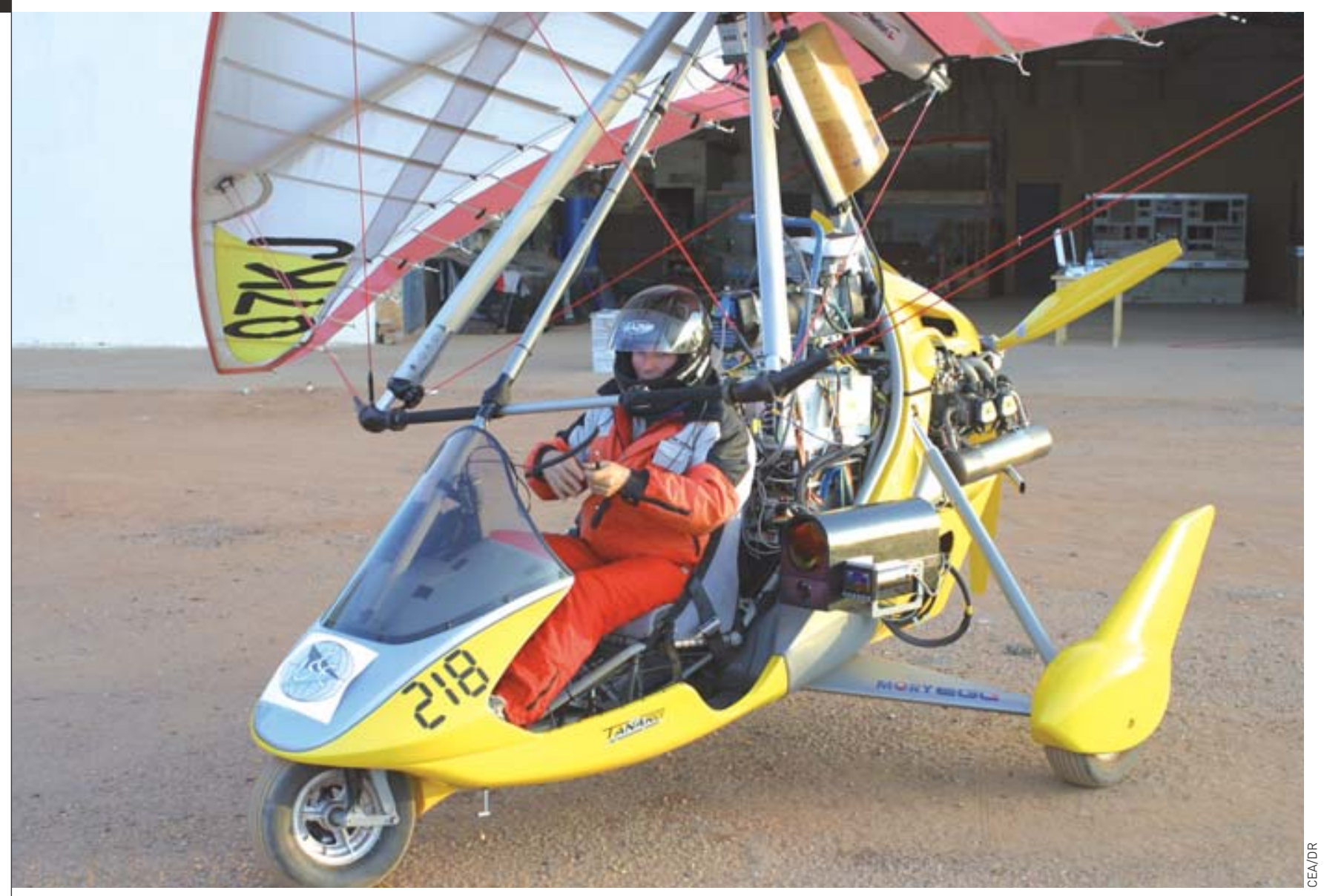

Système lidar embarqué sur un ULM durant la campagne internationale nommée Analyse multidisciplinaire de la mousson africaine (AMMA).

deux fois plus faible qu'à Paris. Celle-ci diminue avec l'altitude jusqu'au sommet de la couche limite planétaire. Cette diminution ne provient pas de la présence plus importante de polluants en altitude. Elle découle de la propriété des particules de pollutions qui peuvent permettre la condensation de vapeur d'eau quand

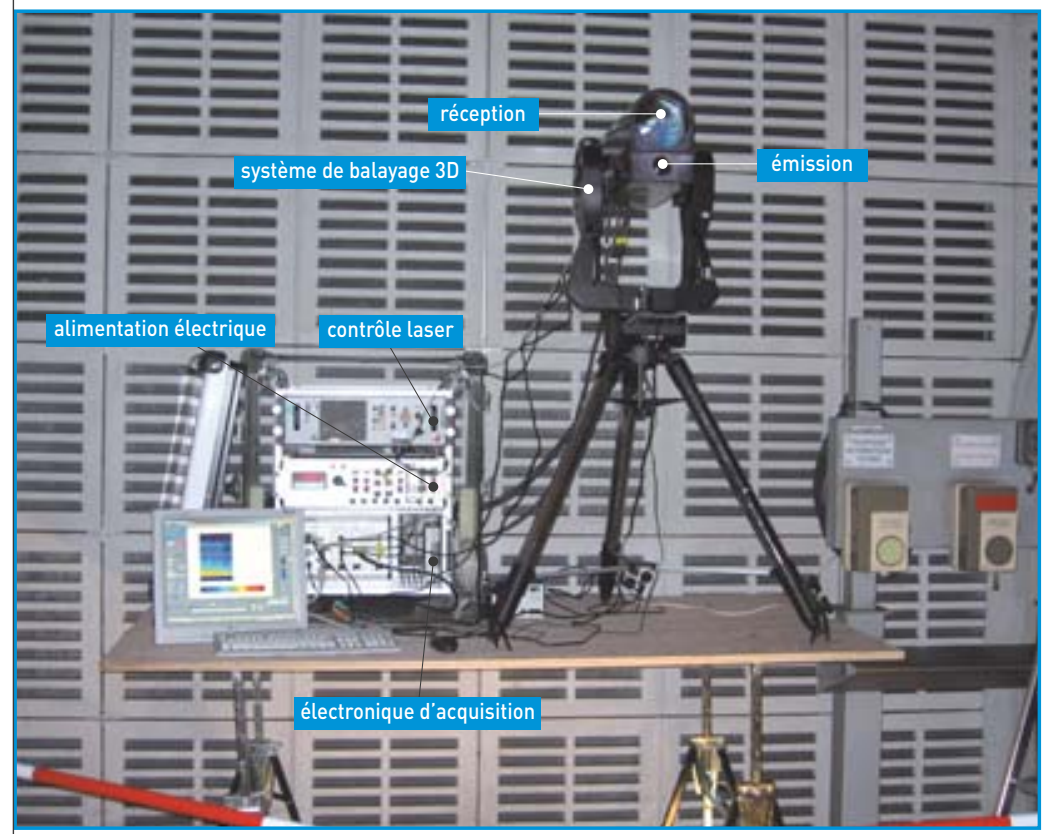

Figure 3 .

Système lidar en fonctionnement pour des études sur la pollution particulaire en gare souterraine avec la SNCF et la RATP. les masses d'air sont humides, un cas fréquent à Pékin. La prudence exige donc de prendre garde aux impressions visuelles pour juger de la pollution car, un ciel "laiteux" ne signifie pas forcément une pollution accrue. Néanmoins, il demeure possible d'affirmer, sans risque, que l'air de Pékin s'avère plus pollué que celui de Paris. À ceci près que Paris et sa banlieue ne comptent qu'environ 12 millions d'habitants contre 17 millions pour la grande mégapole chinoise.

\section{Le lidar pour surveiller l'air intérieur}

Le système lidar développé par le CEA et le CNRS permet également de surveiller la répartition spatiotemporelle des aérosols dans des milieux confinés comme les gares souterraines de la RATP ou de la SNCF. Une expérience conduite gare du Nord, à Paris, a démontré l'intérêt d'un tel instrument de mesure pour évaluer l'influence du passage des trains et des différents types de trafic sur la qualité de l'air intérieur des gares souterraines (figure 3). Depuis, des développements instrumentaux spécifiques sont envisagés, toujours basés sur la technologie lidar.

\section{Un système embarqué pour la surveillance du climat}

En janvier 2006, le système lidar a été embarqué à bord d'un ULM pour survoler la région sahélienne, entre Niamey (Niger) et la frontière du Bénin, afin de mesurer la répartition verticale des particules 


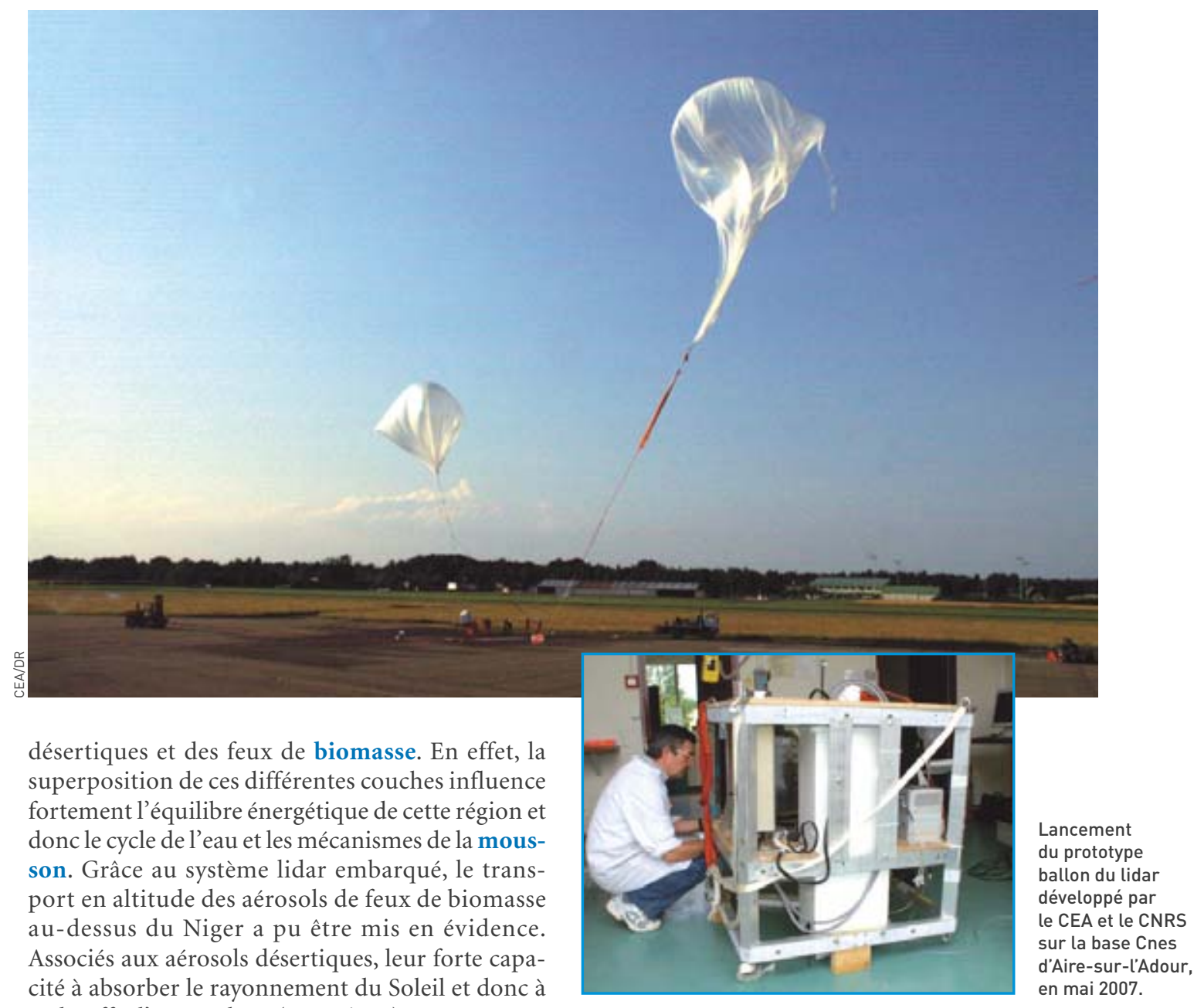
réchauffer l'atmosphère ( $2 \mathrm{~K} /$ jour $)$ tout en créant un déficit énergétique à la surface $\left(\sim-20 \mathrm{~W} \cdot \mathrm{m}^{-2}\right)$ a été démontrée.

\section{Une application pour l'échelle globale}

Des applications pionnières sont nées avec l'arrivé de la nouvelle génération d'instruments spatiaux en orbite autour de la Terre. Il sera désormais possible de suivre l'évolution de certains polluants à l'échelle de la planète grâce à l'observation spatiale. Par exemple, le contenu en ozone de la troposphère est accessible à partir de l'espace. Néanmoins, il demeure difficile d'accéder aux basses couches, "celles où nous vivons”. D'où, l'idée de coupler un système lidar à de nouveaux instruments d'observation comme l'Interféromètre atmosphérique de sondage infrarouge (IASI), actuellement en orbite à bord du satellite européen MetOp. Avec le soutien du Centrenational d'études spatiales (Cnes), un nouveau prototype lidar a été développé. Il peut voler jusqu'à $35 \mathrm{~km}$ d'altitude pour étudier la faisabilité de cette nouvelle configuration instrumentale. Effectués, en mai 2007, sur la base d'Aire-sur-l'Adour du Cnes, les premiers essais ont été un succès.

\section{La qualité de l'air durant les Jeux olympiques 2008}

Après la démonstration réalisée à Pékin, la société Leosphere a développé une collaboration plus approfondie avec la Chine. Celle-ci s'est d'abord concré-

tisée par la vente de deux systèmes commerciaux du lidar. Ces instruments ont été implantés dans la capitale chinoise pour surveiller la qualité de l'air durant les Jeux olympiques de 2008. Ensuite, une coopération a été instaurée avec l'Institut de physique atmosphérique de Pékin et le Centre de surveillance de la qualité de l'air de cette ville. À la suite de quoi, il a été procédé à l'intégration des données lidar dans le système de modélisation de la qualité de l'air, opérationnel pendant les Jeux olympiques. Avec trois cents associations spécialisées dans la qualité de l'air, la Chine offre un vrai potentiel pour le système lidar développé par le CEA et le CNRS. C'est la société Leosphere qui a su conduire cette valorisation au niveau international. D'ores et déjà, des systèmes lidar ont été commercialisés en Angleterre, aux ÉtatsUnis, aux Pays-Bas, en Chine, au Japon et bien sûr en France pour équiper Météo France.

\section{$>$ Patrick Chazette}

Laboratoire des sciences du climat et de l'environnement (LSCE) Institut Pierre-Simon Laplace

Direction des sciences de la matière CEA Centre de Saclay (Orme des Merisiers)

Cet article est dédié à Pierre Couvert qui a joué un rôle majeur dans la mise en opération du système Lauva par ses qualités scientifiques et humaines. Aujourd'hui disparu, la photo de la page 76 le montrant entouré de ses étudiants le rappelle à notre souvenir. 